\title{
Magnetic and Magnetoelectric Effects within a Sandwich Structure with the Parabolic Quantum Well
}

\author{
W. GRUHN \\ Institute of Physics, JD University \\ Al. Armii Krajowej 13/15, 42-200 Częstochowa, Poland
}

We study magnetic effects in a trilayer formed of magnetic layers separated by a spacer with a parabolic potential profile. The focus is on mechanisms of indirect magnetic interactions within the spacer. We show existence of magnetic oscillations and novel type of magnetoelectric effect.

PACS numbers: 75.70.Cn, 75.50.Pp

\section{Introduction}

The semimagnetic semiconductors form a variety of magnetic system with metallic electrical properties [1]. The most promising appear the parabolic quantum well (PQW) systems, which have the ability to absorb light only at the bare harmonic-oscillator frequency irrespective of the electron-electron interaction or the number of electrons in the well. From this reason the magnetic PQW systems are interesting as building blocks spintronic devices. The aim of the paper is to give analytical description of magnetic properties of the sandwich structure having a central PQW layer. In metallic systems conventional calculation of the indirect exchange integrals assumes uniform density and parabolic dispersion of the free charge carriers $[2,3]$. However, in the case of PQW structure the confinement within quantum well makes invalid. Therefore, there is only limited progress in description of magnetic interactions in the nonsquare-QW superlattices [4-7]. Due to the doping (by the transition metal (TM) or rare-earth (RE) metal ions) the semimagnetic semiconductors form a model diluted magnetic system with metallic electrical properties. Provided that the concentration of the free carriers $p$ exceeds critical $p>p_{\mathrm{c}} \approx 3 \times 10^{20} \mathrm{~cm}^{-3}$ the indirect interaction (Ruderman-KittelKasuya-Yosida, RKKY) in a semimagnetic semiconductor system dominates the other mechanisms of magnetic interionic coupling.

\section{Theory}

Assuming that the " $z$ " is the growth direction of a PQW, the Hamiltonian that describes the electronic structure within the envelope function formalism and 
effective mass approximation is given by [1]

$$
H=-\frac{\hbar^{2}}{2 m} \nabla^{2}+\frac{K z^{2}}{2}+E_{0} .
$$

Here, $E_{0}$ and $m$ represent the band gap energy and the effective mass at the center of the PQW, respectively. $K$ is the curvature of the parabolic potential profile, which is assumed to be infinitely high. In view of Hamiltonian (1) the electronic (hole) energy states within the PQW are those of the standard harmonic oscillator. Let us focus our attention on the single band model, with the PQW electron/hole spectrum $\varepsilon_{k, n}=\hbar^{2} k^{2} /(2 m)+\hbar \omega_{\mathrm{D}}(n+1 / 2)-\mu[1]$, where $\mu$ is the Fermi energy. Perturbative calculations of the RKKY exchange integral in the PQW system which account for this spectrum give us [6]:

$$
\chi(r) \approx \frac{\chi_{0}}{r^{d}} r^{2}\left[J_{d / 2-1}(x) Y_{d / 2-1}(x)+J_{d / 2}(x) Y_{d / 2}(x)\right],
$$

with $Y_{\nu}(x)$ being the Neumann function [6] and $d=4$ is the effective spectral dimension of the PQW system. It is worthwhile to mention here that in general in the case of quasi-2D electron gas systems the effective spectral dimension can take any value from the $1 \leq d \leq 4$ range [8].

It can be easily seen that energy spectrum bears, to some extent, formal resemblance to the picture observed in the de Haas-van Alfven (dHvA) effect, the ladder of equidistant states superimposed onto continuous spectrum. Characteristic feature of all systems that exhibit discrete type of spectrum are the electron density oscillations that occur when an oscillator level crosses the Fermi energy. In conventional $\mathrm{dHvA}$ effect the oscillator energy shift is generated by the variable external magnetic field which determines the $\omega_{\mathrm{H}}$. We will show that in our system this role is played by the parameter $D$, where $D$ is the PQW thickness. To prove that let us relate the value of eigenfrequency $\omega_{\mathrm{D}}$ to the material constants of the heterocomponents $\mathrm{A}$ and $\mathrm{B}$ that form the $\mathrm{PQW}$. The quantum wells with parabolic potential profile are fabricated via continuous $\mathrm{A}_{x} \mathrm{~B}_{1-x}$ variation of heterocomponents along the " $z$ " direction. Let us suppose that the conduction band edges of the heterocomponents are given by $\mathrm{A} \rightarrow V_{\mathrm{A}}$ and $\mathrm{B} \rightarrow V_{\mathrm{B}}$, then the height of the PQW potential barrier can be expressed as $\Delta V=V_{\mathrm{A}}-V_{\mathrm{B}}$. Having that we can determine the constant $K$ in Eq. (1) from the condition $K D^{2} / 8=\Delta V$. Consequently we can calculate the eigenfrequency $\omega_{\mathrm{D}}$ as $\omega_{\mathrm{D}}^{2}=8 \Delta V /\left(m^{*} D^{2}\right)$. In principle this expression resembles the eigenspectrum of free electrons being subject to the external magnetic field $B=\left(2 m^{2} c^{2} \Delta V\right)^{1 / 2} /\left(e^{2} m^{*} D^{2}\right)^{1 / 2}$. Assuming the canonical ensemble the thermodynamical potential $\Omega$ of the unbound (mobile) charge carriers within the PQW can be found [9]. At $T=0$ the contribution $\delta \Omega$ of the electrons that have momenta within the $k$ and $k+\mathrm{d} k(k=|\boldsymbol{k}|)$ reads

$$
\delta \Omega=k \mathrm{~d} k \frac{\beta m^{2} c}{\pi m^{*} D^{2}}\left[(X-n-1 / 2)^{2}-(X-n-1 / 2)+1 / 6\right],
$$

where $(n+1 / 2) \leq X \leq(n+3 / 2)$ and $X=A /(2 \pi m)\left(m^{*} / \Delta V\right)^{1 / 2} D$, if $X$ is outside this region then in formula (3) $n$ should be changed by one i.e., $n \rightarrow n+1$ [10]. 
This means that the thermodynamical potential shows regular oscillations as the function of PQW layer thickness $D$. In the case of arbitrary temperature $T$ the potential $\Omega$ can be estimated with the help of the Poisson formula and takes the form [9]: $\Omega=\Omega_{0}+\hat{\Omega}$, where the $\Omega_{0}$ is the classical part of the potential $\Omega$ which does not oscillate as the function of $D$ [9]. Contrary to $\Omega_{0}$ the $\hat{\Omega}$ term behaves as [9]:

$$
\hat{\Omega}=\hat{\Omega}_{0}(D)+2 \sum_{k} \sum_{r=1}^{\infty} A_{k}^{r} \cos \left(r f D+\phi_{k}^{r}\right) .
$$

The free energy of the system defined as $\hat{F}=\hat{\Omega}-1 /(2 \varrho)(\partial \hat{\Omega} / \partial \mu)$ also shows periodical oscillation as a function of $D$. Consequently if we account for the external magnetic field $H$ the effective magnetization within the layer can be estimated as $M=-(\partial F / \partial H)_{T}$, shows oscillatory behaviour as the function of the PQW thickness. The density oscillations can be attributed to changes of the electron density at the Fermi level when one of the $h \omega_{D}(n+1 / 2)$ levels crosses the Fermi energy. The oscillatory behaviour can be attributed to the change of electron population at Fermi energy. There is another effect that influences the spin polarization of the electrons confined within the PQW. Let us suppose that the magnetizations of the adjacent uniform layers are oriented parallelly, then all energy levels are split by the $g \sigma \mu_{\mathrm{B}} B$ term. Let us suppose that we change the PQW thickness then the discrete energy levels $\varepsilon_{n, \uparrow}$ and $\varepsilon_{n, \downarrow}$ of the spin up and spin down electrons, cross the Fermi level at different value of $D$. This produces additional oscillatory contribution of the PQW magnetization which varies between some $M_{\max }$ and zero. In the conventional (thin) quantum wells with the rectangular potential profiles the discrete energy levels (or centers of minibands) behave as $\varepsilon_{k} \propto k^{2}$. Since $k \propto D^{-1}$ we have $\varepsilon_{k} \propto D^{-2}$, while in the case of our system we have $\varepsilon_{n} \propto \omega_{D} \propto D^{-1}$. Thus, one would expect different envelope function of oscillations when compared to the rectangular systems.

Let us suppose that the PQW is subject to the external uniform electric field $E$. The Hamiltonian $H^{\prime}$ of the charge carriers differs from the Hamiltonian (1) by the term which comes from the external electric field,i.e., $H^{\prime}=H+e E z$. Formally, Hamiltonian $H^{\prime}$ represents the displaced harmonic oscillator

$$
H^{\prime}=E_{0}^{\prime}+\frac{\hbar^{2}}{2 m} \nabla^{2}+\frac{K(z \pm a)^{2}}{2} .
$$

The sign in the third term in Eq. (5) depends on the direction of the applied external field, while $E_{0}^{\prime}=E_{0}-e^{2} E^{2} / K$ and $a=e E / K$. From Eq. (5) results that in the presence of the external electric field the shape of the quantum well remains parabolic, however, the position of the centre of the well is shifted to the right or left with respect to the case $\boldsymbol{E}=0$. Moreover, the minimum of the parabolic potential well is shifted down (independently on the direction of $\boldsymbol{E}$ ) by the value $\Delta E_{0}=(e E)^{2} / K$. Consequently the discrete oscillator levels are described by the same frequency $\omega_{D}$ as in the case $\boldsymbol{E}=0$, but all energy levels are shifted down by 
the value $\Delta \varepsilon_{n k}=(e E)^{2} / K$. This means that with the change of external field the positions of the discrete levels with respect to the Fermi energy are changed by the external electric fields. As we have shown, such change results in the oscillation of the magnetization within the PQW. Generally, any induced magnetization under action of external electric field is called as magnetoelectric effect. Magnetoelectric effect is observed in many multifunctional composites [11], however, its origin is different from that of our system.

\section{Conclusions}

In conclusion, we have proved that the free energy of the system (and as result of that other characteristics) show oscillatory behaviour as the PQW thickness $D$ is changed. The most interesting effect arises when there arises external electric field oriented along the PQW growth direction. The shift of energy levels, being a function of the external field can modify the spin polarization of the unbound charge carriers within PQW. From the formal point of view this is a new mechanism of magnetoelectric effect. In real systems this effect can be used to manipulate from outside the properties of $\mathrm{PQW}$ based multilayer systems.

\section{References}

[1] W. Geisselbrecht, A. Masten, O. Gräbner, M. Forkel, G.H. Döhler, C. Campman, A.C. Gossard, Superlattices Microstructures 23, 93 (1998).

[2] T. Dietl, A. Haury, Y. M. d'Aubigné, Phys. Rev. B 55, R3347 (1997).

[3] V.K. Dugaev, V.I. Litvinov, W. Dobrowolski, T. Story, Solid State Commun. 110, 351 (1999).

[4] Z. Bak, Solid State Commun 118, 43 (2001).

[5] Z. Bak, Czech. J. Phys. 52, A25 (2002).

[6] Z. Bak, J. Jaroszewicz, W. Gruhn, J. Magn. Magn. Mater. 213, 340 (2000).

[7] M. Sawicki, T. Dietl, G. Karczewski, T. Wojtowicz, J. Kossut, T. Skośkiewicz, Acta Phys. Pol. A 90, 919 (1996).

[8] Z. Bak, Phys. Rev. B 68, 064511 (2003).

[9] A.S. Alexandrov, A.M. Bratkovsky, Phys. Rev. B 69, 167401 (2004).

[10] D. Shoenberg, Magnetic Oscillations in Metals, Mir, Moscow 1986, p. 64.

[11] G. Srinivasan, C.P. De Vreugd, V.M. Laletin, N. Paddubnaya, M.I. Bichurin, V.M. Petrov, D.A. Filippov, Phys. Rev. B 71, 184423 (2005). 